\title{
Characteristics of nanosized ruthenium metal by chemical reduction method
}

\author{
Der-Shing Lee *, Tsung-Kwei Liu \\ Department of Geosciences, National Taiwan University, Taipei, 106 Taiwan, ROC \\ Received 11 December 2001; received in revised form 9 May 2002
}

\begin{abstract}
Nano-sized Ru metals have been prepared by the chemical reduction of ruthenium chloride and ruthenium hydroxide. Sodium borohydride was used as a reducing agent. The samples have been characterized by elemental analysis, $\mathrm{X}$-ray diffraction, differential scanning calorimetry, and transmission electron microscopy. The preparation method greatly affects the composition and surface area of the material. All the samples show nanosized particles. However, samples prepared by reduction of ruthenium hydroxide had a lower surface area and larger particle size than those prepared by reduction of ruthenium chloride. Residual amount of boron was present in the samples. The samples demonstrate amorphous structure.
\end{abstract}

(C) 2002 Elsevier Science B.V. All rights reserved.

\section{Introduction}

Nanosized materials have attracted extensive interest in recent years, [1-12] due to their unique isotropic, structural and chemical properties. Previous investigators have reported that nanosized materials can possess novel physical, mechanical, and chemical properties, particularly for catalytic and magnetic recording applications. [1,13,14] There are two major techniques used for producing the nanosized amorphous alloy. One is the chemical technique, the other is the physical

\footnotetext{
${ }^{*}$ Corresponding author. Address: Department of Geosciences, National Taiwan University, 245 Choushan Road, Taipei, Taiwan.

E-mail address: ipo02022@mail.moeaipo.org.tw (D.-S. Lee).
}

technique. The chemical technique includes chemical reduction and the sol-gel process. The physical technique includes deposition from vapor, etc. Of relevant interest when using nanosized amorphous metal is how to decrease the particle size of the material. Generally, the particle size of nanosized metal prepared by vapor and sputter deposition, and melt-quenching is rather large. [15] Nanosized metals can also be produced by sol-gel method using metal alkoxides as the starting materials. However, metal alkoxides are expensive and the strict preparation conditions are required. Nanosized metals produced by chemical reduction have received increasing attention in recent years. The nanosized metal particles obtained by chemical reduction have the obvious merits of a smaller particle size than those prepared by vapor and sputtering deposition and melt-quenching [16,17]. 
The preparation condition is also not so strict as that of sol-gel method. Therefore, the cost would be lower. In addition, it can be produced in continuous process.

In the present study, a series of nanosized ruthenium metals were prepared by two chemical reduction methods. In the first method, ruthenium chloride was reduced by sodium borohydride. In the second method, ruthenium chloride was converted to hydroxide and then reduced by sodium borohydride. The objective of this study was to investigate the effects of the preparation method on the characteristics of the materials. The materials were characterized by elemental analysis, X-ray diffraction (XRD), nitrogen sorption, differential scanning calorimetry (DSC) and transmission electron microscopy (TEM).

\section{Experimental}

\subsection{Chemicals}

Ruthenium (III) chloride hydrate ( $>99.5 \%$ ), sodium hydroxide $(>95 \%)$ and sodium borohydride $(>98 \%$ ) were used.

\subsection{Sample preparation}

Ruthenium samples were prepared by two methods: (i) direct reduction method. Ruthenium chloride was reduced directly with sodium borohydride. An excess amount of sodium borohydride was added drop by drop to the aqueous solution of ruthenium chloride under ultrasonic agitation. The precipitate was collected on a ceramic filter and washed thoroughly with distilled water. The sample was then rinsed with acetone, filtered and dried at room temperature. (ii) Hydroxide method. Ruthenium chloride was converted to hydroxide and then reduced. An excess amount of aqueous sodium hydroxide solution (30 $\mathrm{wt} \% \mathrm{NaOH})$ was added to the solution of ruthenium chloride to form ruthenium hydroxide. After aging at $70{ }^{\circ} \mathrm{C}$ for $1 \mathrm{~h}$, the solution was cooled to to room temperature. Excess amount of sodium borohydride was added drop by drop to the solution under ultrasonic agitation.

\subsection{Elemental analysis}

Inductively coupled plasma-atomic emission spectroscopy (ICP-AES) was used to determine the composition of the samples. A sequential ICP spectrometer was used to determine the contents of $\mathrm{Ru}$ and $\mathrm{B}$. Weighed samples were dissolved in hydrochloric acid and diluted with distilled water to a concentration within the calibration range of each element. The standard solutions were diluted and used to establish the calibration curves. The wavelengths $(\mathrm{nm})$ used for elemental analysis were 240.272 and 249.773 for $\mathrm{Ru}$ and $\mathrm{B}$, respectively.

\subsection{X-ray diffraction}

XRD patterns of the samples were recorded on a diffractometer with nickel-filtered $\mathrm{CuK}_{\alpha}$ radiation. The X-ray tube was operated at $40 \mathrm{kV}$ and 30 $\mathrm{mA}$. Samples were scanned over the range $2 \theta=$ $10-80^{\circ}$ at a rate of $0.05^{\circ} \mathrm{min}^{-1}$. A scan of silica was used as a standard.

\subsection{Nitrogen sorption}

The nitrogen adsorption isotherms and specific surface areas were determined by nitrogen physisorption with the BET method at liquid nitrogen temperature using an automatic analyzer. Prior to measurement, the samples were degassed at $323 \mathrm{~K}$ for $12 \mathrm{~h}$. The final pressure was $\approx 0.1 \mathrm{~Pa}$ in the closed system for at least $1 \mathrm{~min}$. BET surface areas were calculated in a relative pressure range between 0.01 and 0.2 assuming a cross-section area $0.162 \mathrm{~nm}^{2}$ for the nitrogen molecules.

\subsection{Differential scanning calorimetry}

DSC was conducted under nitrogen $(99.99 \%)$ atmosphere. The sample was scanned over the range $50-550{ }^{\circ} \mathrm{C}$ at a rate of $10{ }^{\circ} \mathrm{C} / \mathrm{min}$ to investigate the crystalline processes of the amorphous structure.

\subsection{Transmission electron microscopy}

TEM was used to investigate the morphologies and particle sizes of the ruthenium samples. Im- 
ages were taken on transmission electron microscope operated at $200 \mathrm{kV}$. A small amount of the sample was put into a sample tube filled with anhydrous alcohol. After agitating the sample under an ultrasonic environment for $30 \mathrm{~min}$, one drop of the dispersed slurry was dipped on a carboncoated copper mesh (300\#).

\section{Results and discussion}

\subsection{Elemental analysis}

The ruthenium metals were prepared by the reduction of either ruthenium chloride or ruthenium hydroxide with an excess amount of sodium borohydride. Table 1 shows the composition of the samples prepared by the reduction of ruthenium chloride. A small amount of boron was found in the samples. The boron content in the solid sample increased with increasing boron content in the mother solution. In addition, the boron content in solid samples prepared by the reduction of ruthenium chloride was greater than in the samples prepared by the reduction of ruthenium hydroxide. No chlorine ion was detected I the samples due to the thorough wash of the samples.

\subsection{X-ray diffraction}

The XRD patterns of the samples as shown in Fig. 1 have a broad peak around $2 \theta=45^{\circ}$. This can be assigned to the amorphous state of

Table 1

The characteristics of the samples

\begin{tabular}{|c|c|c|c|c|}
\hline Sample & $\begin{array}{l}\mathrm{B} / \mathrm{Ru} \text { ratio } \\
\text { in solution }\end{array}$ & $\begin{array}{l}\text { Compo- } \\
\text { sition }\end{array}$ & $\begin{array}{l}\text { BET sur- } \\
\text { face area } \\
\left(\mathrm{m}^{2} / \mathrm{g}\right)\end{array}$ & $\begin{array}{l}\text { Particle } \\
\text { size }(\mathrm{nm})\end{array}$ \\
\hline \multicolumn{5}{|c|}{ Reduction of ruthenium chloride } \\
\hline A-1 & $6 / 1$ & $\mathrm{Ru}_{98.3} \mathrm{~B}_{1.7}$ & 32.9 & 20.4 \\
\hline A-2 & $3 / 1$ & $\mathrm{Ru}_{98.7} \mathrm{~B}_{1.3}$ & 30.7 & 21.8 \\
\hline A-3 & $1 / 1$ & $\mathrm{Ru}_{99.2} \mathrm{~B}_{0.8}$ & 18.6 & 36.0 \\
\hline \multicolumn{5}{|c|}{ Reduction of ruthenium hydroxide } \\
\hline B-1 & $3 / 1$ & $\mathrm{Ru}_{96.2} \mathrm{~B}_{3.8}$ & 18.2 & 36.8 \\
\hline B-2 & $1 / 1$ & $\mathrm{Ru}_{97.4} \mathrm{~B}_{2.6}$ & 14.2 & 47.2 \\
\hline B-3 & $1 / 3$ & $\mathrm{Ru}_{98.1} \mathrm{~B}_{1.9}$ & 10.2 & 65.7 \\
\hline
\end{tabular}

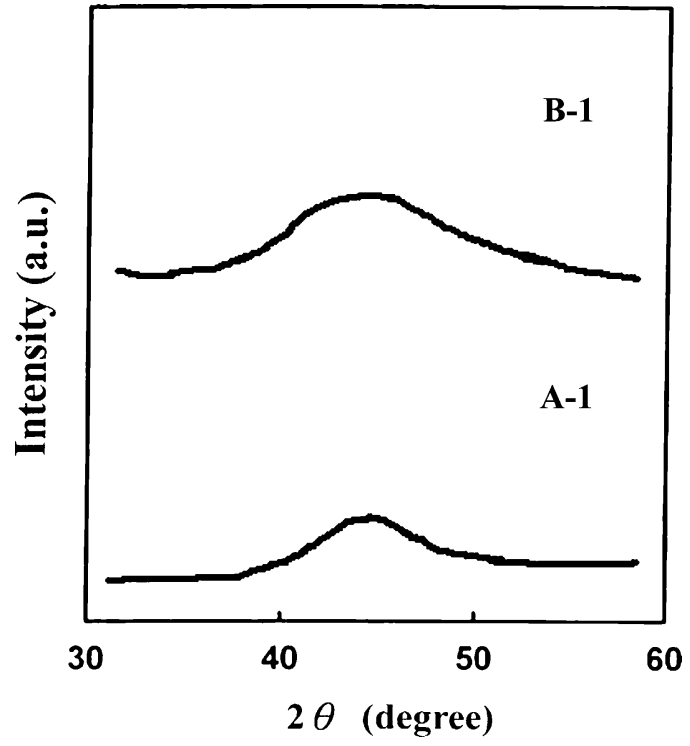

Fig. 1. XRD patterns.

ruthenium. [13,18-20] The XRD patterns did not contain any distinct peaks corresponding to a crystalline phase for all the samples. All the samples demonstrate an amorphous structure.

\subsection{Nitrogen sorption}

Table 1 shows that the samples prepared by the reduction of ruthenium hydroxide had a lower surface area than those prepared by the reduction of ruthenium chloride. Nitrogen sorption results showed that these samples were not porous. Calculation of the particle sizes from the BET surface areas, with the assumption of free spherical structure, yields values of $10-30 \mathrm{~nm}$, which are in good agreement with those from TEM as shown in a later section. Struijk et al. [21] applied the hydroxide method to prepare ruthenium particles. Their catalysts were reduced at a much higher temperature with hydrogen gas $\left(>200{ }^{\circ} \mathrm{C}\right)$. The porous polycrystalline conglomerates obtained had particle sizes of $10-150 \mu \mathrm{m}$. In this study, the samples were reduced at room temperature with sodium borohydride. The above findings reconfirm that high temperature hydrogen reduction results in larger particle size of the catalyst. 


\subsection{Differential scanning calorimetry}

It is evident from DSC results (Fig. 2) that $\mathrm{Ru}$ samples prepared by the reduction of ruthenium chloride (sample A-2) had a smaller particle size than those prepared by the reduction of ruthenium hydroxide (sample B-2). Sample B-2 had a higher thermal stability. The growth of crystalline nuclei in the process of forming a new crystalline phase in an amorphous phase can be divided into two stages: one is the formation of crystalline nuclei, the other is the growth of nuclei. The formation of a new crystalline phase on the surface can reduce the surface energy. It thus appears that the surface energy of smaller particles is higher. The tendency to form a stable crystalline state, hereby decreasing the surface energy on smaller particle surfaces, is therefore stronger. The number of crystalline nuclei formed in smaller particles is much greater, hence the small particles have a lower thermal stability.

\subsection{Transmission electron microscopy}

Distinct differences in the morphologies and particle sizes of the samples are observed in the TEM micrographs. The samples demonstrate a particle-aggregate morphology as shown in Fig. 3. The particle sizes of the samples A-2 and B-2 (diameter range $10-30 \mathrm{~nm}$ ) were determined from TEM micrographs. By assuming that the Ru particles are spherical and non-porous, their average

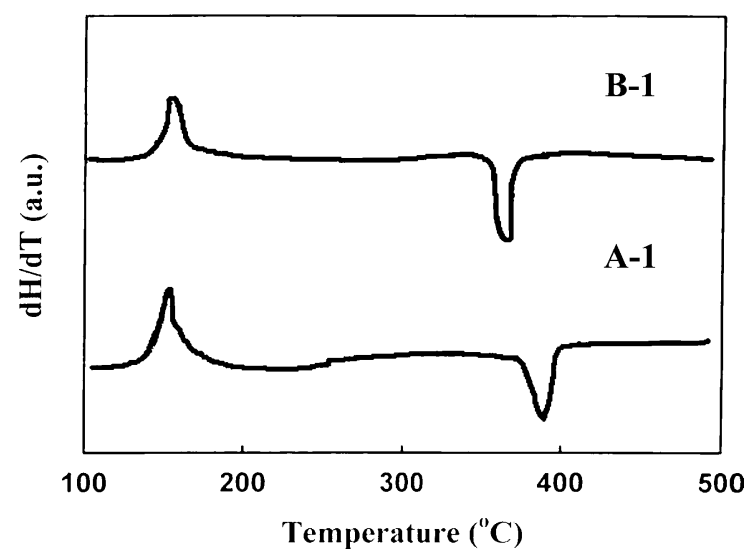

Fig. 2. DSC results.

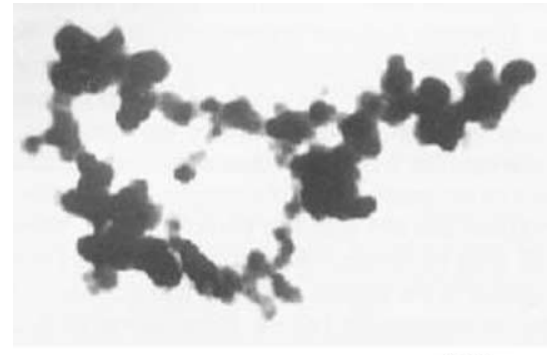

$100 \mathrm{~nm}$

(I)

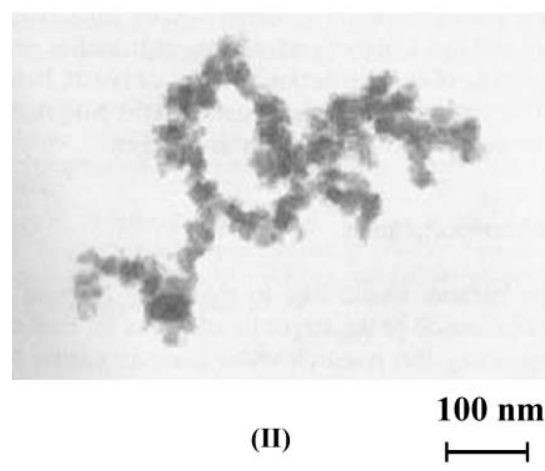

Fig. 3. TEM micrographs: (I) sample A-1 and (II) sample B-1.

sizes can be estimated by the equation $\bar{d}(\mathrm{~nm})=$ $6 / S_{\mathrm{BET}} \rho \times 10^{3}$ where $S_{\mathrm{BET}}$ denotes the surface area and $\rho$ is the density of the metal. The particle sizes from TEM and those calculated from surface area measurements are in good agreement.

\section{Conclusion}

A series of ultrafine $\mathrm{Ru}$ catalysts have been prepared by reduction of ruthenium chloride and ruthenium hydroxide. Sodium borohydride was used as a reducing agent to avoid the agglomeration problem posed by high temperature reduction when using hydrogen gas as a reducing agent. The preparation method has great influence on the composition and surface area of the samples. The samples prepared by the reduction of ruthenium hydroxide have a lower surface area and larger particle size than the samples prepared by the reduction of ruthenium chloride. Residual amount of boron was present in the samples. The samples demonstrate amorphous structure. 


\section{References}

[1] J.V. Wonterghem, S. Morup, J.W. Christion, S. Charles, W.S. Wells, Nature 322 (1986) 622.

[2] A. Corrias, G. Ennas, G. Licheri, G. Marongiu, A. Musinu, G. Paschina, G. Piccaluga, G. Pinna, M. Magini, J. Mater. Sci. Lett. 7 (1988) 407.

[3] S. Linderoth, S. Morup, A. Meagher, J. Larsen, M.D. Bentzon, B.S. Clausen, C.J.W. Koch, S. Wells, S.W. Charles, J. Magn. Magn. Mater. 81 (1989) 138.

[4] S. Linderoth, S. Morup, J. Appl. Phys. 67 (1990) 4472.

[5] D.S. Xue, F.S. Li, R.J. Zhou, J. Mater. Sci. Lett. 9 (1990) 506.

[6] J. Jiang, U. Dezsi, S. Gonser, X. Lin, J. Non-Cryst. Solids 124 (1990) 139.

[7] J. Shen, Y.Z. Hu, L.F. Zhang, Y.Z. Li, Y. Chen, Appl. Phys. Lett. 59 (1991) 545.

[8] J.Y. Shen, Z. Hu, Y.F. Hsia, Appl. Phys. Lett. 59 (1991) 2510.

[9] J. Saida, A. Inoue, T. Masumoto, Mater. Sci. Eng. 133 (1991) 771.
[10] J.F. Deng, H.Y. Chen, J. Mater. Sci. Lett. 12 (1993) 1508.

[11] Z. Hu, J.Y. Shen, Y.N. Fan, Y.F. Hsia, Y. Chen, J. Mater. Sci. Lett. 12 (1993) 1020.

[12] J.Y. Shen, Z. Hu, Q. Zhang, L.F. Zhang, Y. Chen, J. Appl. Phys. 71 (1992) 5217.

[13] H. Yamashita, H. Yoshikawa, T. Funabiki, S. Yoshida, J. Chem. Soc., Faraday Trans. I 82 (1986) 1771.

[14] Y. Okamoto, Y. Nitta, T. Imanaka, S. Teranishi, J. Chem. Soc., Faraday Trans. I 75 (1979) 2027.

[15] J. Deng, X. Zhang, E. Min, Appl. Catal. 37 (1988) 339.

[16] S.P. Lee, Y.W. Chen, Ind. Eng. Chem. Res. 38 (1999) 2548.

[17] S.P. Lee, Y.W. Chen, J. Mol. Catal. 152 (2000) 213.

[18] J. Deng, J. Yang, S. Sheng, H. Chen, G. Xiong, J. Catal. 150 (1994) 434.

[19] Y. Okamoto, Y. Nitta, T. Imanaka, S. Teranishi, J. Chem. Soc. Faraday Trans. I 76 (1980) 998.

[20] Y. Okamoto, Y. Nitta, T. Imanaka, S. Teranishi, J. Catal. 64 (1980) 397.

[21] J. Struijk, R. Moere, T. van der Kamp, J.J.F. Scholter, Appl. Catal. A 89 (1992) 77. 